\title{
Flunitrazepam Measurement
}

National Cancer Institute

\section{Source}

National Cancer Institute. Flunitrazepam Measurement. NCI Thesaurus. Code C139081.

The determination of the amount of flunitrazepam present in a sample. 